\title{
Microvascular decompression for tinnitus: systematic review
}

\author{
Brenton Nash, BA, ${ }^{3}$ Matthew L. Carlson, MD, ${ }^{1,2}$ and Jamie J. Van Gompel, MD ${ }^{1,2}$ \\ Departments of ${ }^{1}$ Neurological Surgery and ${ }^{2}$ Otolaryngology, Mayo Clinic School of Medicine, Rochester, Minnesota; and \\ ${ }^{3}$ College of Medicine, University of Vermont, Burlington, Vermont
}

\begin{abstract}
OBJECTIVE The objective of this study was to examine operative outcomes in cases of microvascular decompression (MVD) of cranial nerve (CN) VIII for tinnitus through a critical review of the literature.

METHODS Forty-three English-language articles were gathered from PubMed and analyzed. In this review, two different case types were distinguished: 1) tinnitus-only symptomatology, which was defined as a patient with tinnitus with or without sensorineural hearing loss; and 2) mixed symptomatology, which was defined as tinnitus with symptoms of other $\mathrm{CN}$ dysfunction. This review reports outcomes of those with tinnitus-only symptoms.

RESULTS Forty-three tinnitus-only cases were found in the literature with a $60 \%$ positive outcome rate following MVD. Analysis revealed a 5 -year cutoff of preoperative symptom duration before which a good outcome can be predicted with $78.6 \%$ sensitivity, and after which a poor outcome can be predicted with $80 \%$ specificity.
\end{abstract}

CONCLUSIONS As the $60 \%$ success rate is more promising than several other therapeutic options open to the chronic tinnitus sufferer, future research into this field is warranted.

https://thejns.org/doi/abs/10.3171/2016.2.JNS152913

KEY WORDS microvascular decompression; tinnitus; retrosigmoid craniotomy; functional neurosurgery

$\mathrm{I}$ N 1959, W. James Gardner performed the first microvascular decompression (MVD) without concomitant neurolysis for trigeminal neuralgia (TN) to good effect. ${ }^{21}$ Microvascular decompression for TN and other neurovascular compression (NVC) syndromes was subsequently refined and popularized by Peter Jannetta. ${ }^{12}$ The success of the procedure lent credence to Walter Dandy's observations made decades prior that NVC of cranial nerves (CNs) may lead to hyperactive disorders. ${ }^{6}$ With these etiological assumptions supported by the success of MVD for TN, investigators began to speculate as to NVC's role in other paroxysmal, hyperactive cranial neuralgias. As the next natural progression, they then began to explore the applicability of MVD. ${ }^{24}$

The first disorder to which MVD was expanded was hemifacial spasm (HFS) by Gardner himself. ${ }^{21}$ In 1975, Jannetta attempted to explain hyperactive CN VIII symptoms such as intractable tinnitus and/or vertigo with the same NVC pathophysiology. Subsequently, Jannetta performed MVDs on 8 patients for said indications. ${ }^{12}$ This is, to the authors' knowledge, the first example of MVD without concomitant neurolysis being performed on $\mathrm{CN}$ VIII. Jannetta's work with these initial 8 patients laid the groundwork for a whole field of neurosurgical research and countless operative interventions.

More than 50 million people in the US report having symptoms of tinnitus, which can be extrapolated to an estimated $10 \%-15 \%$ prevalence among the adult population. ${ }^{42}$ For some, tinnitus may be regarded as a minor nuisance; however, for many, tinnitus results in a substantial quality of life reduction and may exacerbate conditions of depression and anxiety. Despite significant research and clinical attention, the underlying pathophysiology and treatment of tinnitus remain poorly defined. In most cases, an underlying cause other than coexisting sensorineural hearing loss (SNHL) is not identified. Less commonly, secondary tinnitus may result from conditions such as Ménière's disease, otosclerosis, or vestibular schwannoma. The American Academy of Otolaryngology-Head and Neck Surgery (AAO-HNS) recently issued a clinical practice guideline

ABBREVIATIONS AAO-HNS = American Academy of Otolaryngology-Head and Neck Surgery; $\mathrm{CBT}=$ cognitive behavioral therapy; $\mathrm{CN}=\mathrm{cranial}$ nerve; $\mathrm{CPA}=\mathrm{cerebel}$ lopontine angle; FIESTA = fast imaging employing steady-state acquisition; HFS = hemifacial spasm; MVD = microvascular decompression; NVC = neurovascular compression; $\mathrm{ROC}=$ receiver operating characteristic; $\mathrm{SNHL}=$ sensorineural hearing loss; $\mathrm{TN}=$ trigeminal neuralgia.

SUBMITTED December 14, 2015. ACCEPTED February 10, 2016.

INCLUDE WHEN CITING Published online May 20, 2016; DOI: 10.3171/2016.2.JNS152913. 
for tinnitus in 2014. ${ }^{43}$ Based on analysis, hearing aid evaluation and cognitive behavioral therapy (CBT) were recommended; however, these guidelines recommended against employment of medical therapies, dietary supplements, or transcranial magnetic stimulation for most cases of bothersome tinnitus. Use of posterior fossa MVD in the treatment algorithm for tinnitus was not addressed. ${ }^{43}$

In this paper we present a systematic review of MVD for tinnitus. Case presentations fit 1 of 2 patterns: "tinnitus-only" symptoms or "mixed" symptoms. Tinnitus-only symptoms refer to cases exhibiting cochlear dysfunction (tinnitus with or without SNHL) in the absence of other clinically relevant $\mathrm{CN}$ involvement. Conversely, mixed symptom presentations exhibit vestibular or other $\mathrm{CN}$ dysfunction with simultaneous cochlear dysfunction. Other $\mathrm{CN}$ involvement includes but is not limited to vestibular symptoms (vertigo or disequilibrium), HFS, TN, and geniculate neuralgia.

Tremendous heterogeneity in reporting on this subject exists in the literature. One study might analyze the operative outcomes of MVD for tinnitus in mixed-symptom cases. Yet another study may index patients with tinnitusonly symptoms. These two vastly different study types often cross-reference one another and treat their results as comparable. However, it is likely that this assumption is flawed. Mixed-symptom cases may be more easily diagnosed as a number of different $\mathrm{CN}$ symptoms may form a readily localized constellation of symptoms. For example, it may be more apparent to a clinician that a patient with both HFS and incapacitating tinnitus has an aberrant vascular loop compressing both CN VIII and CN VII. Conversely, it may be more difficult to diagnose NVC based upon tinnitus and SNHL, a symptom constellation that has a long differential diagnosis. This point is partially supported in the literature: studies have shown that tinnitus occurring with HFS is resolved/improved by MVD in $84.6 \%$ of cases. ${ }^{38,39}$ The purpose of this article is to provide the most complete collection of tinnitus-only case data and compare these results with assertions made in the larger, heterogeneous literature of MVD for tinnitus.

\section{Methods \\ Literature Search}

A search of the English-language literature was performed using the PubMed online database with the following search parameters: "tinnitus" AND "neurovascular compression" OR "microvascular compression" AND "microvascular decompression" OR "neurovascular decompression" AND "cranial nerve eight" OR "cochleovestibular nerve." This query returned 13,797 results, which were then sorted according to relevance. From this list, full-text versions of articles deemed relevant were obtained through institutional resources. Relevant articles were considered those with titles or abstracts referencing NVC of CN VIII, tinnitus, and/or MVD. Additional articles were also identified from the bibliographies of relevant sources. This cross-referencing method was also used to ensure completeness in the present review.

The initial search yielded 43 articles. All articles were run through a 2-tier analysis. The first tier of inclusion cri- teria required each article to report 1 or more cases of NVC of CN VIII. In addition, it was required that each case present with tinnitus-only symptoms and, subsequently, undergo MVD. This first-tier analysis is represented in Fig. 1. Nine of the initial 43 articles emerged from tier-one analysis. ${ }^{5,10,16,18,22,37,41,44,46}$ Six articles were excluded as they presented cases of NVC of CN VIII in which nonoperative or unspecified management was pursued. , $^{1,18-20,28,32}$ Fourteen articles were excluded as cases included mixed-symptom presentations. . $^{-4,15,21,25,26,28-31,38-40,45}$ Six articles were excluded because case demographic information and/or results were not stratified by presentation; tinnitus-only cases were not delineated appropriately from mixed-symptom cases. ${ }^{9,12,14,27,34,47}$ Five articles were excluded for suspicion of duplicating case data from prior works by 1 or more of the same authors. ${ }^{7,8,11,13,35,36}$ And 3 articles were excluded for being review articles containing no novel case data. ${ }^{14,23,42}$

From the 9 tier-one articles, 106 cases were considered for inclusion in the final analysis. Sixty-three cases were eventually excluded as their presentations included symptoms of vestibular and/or other CN dysfunction (Fig. 2). The 43 remaining cases were included in the final analysis (Table 1).

\section{Statistical Analysis}

For the purpose of all statistical tests, the operative outcomes were condensed to a dichotomous scale of "good outcome" (resolved and improved) versus "poor outcome" (no change and worse). All analyses were performed with SPSS (IBM).

Based upon the data presented in Table 1, the relationships between various case variables were compared with operative outcome. Chi-square analysis was performed to assess the relationship between patient sex and operative outcome. Additionally, lambda was calculated to assess the relationship between the culprit vessel compressing CN VIII and operative outcome, with the latter treated as the dependent variable. Lastly, discriminant analysis was performed and a receiver operating characteristic (ROC) curve created to determine the relationship between preoperative duration of tinnitus and operative outcome.

\section{Results}

The final analysis consisted of 43 unique cases, including 22 men and 21 women ranging in age from 29 to 76 years (mean 51 years; Table 1). On average, patients experienced tinnitus symptoms for 4 years prior to MVD (range 5 months to 25 years). Nine patients experienced complete resolution of their tinnitus following MVD, 17 patients experienced improvement of their tinnitus symptoms (defined differently between studies, and for the purposes of the present review denotes any diminution of tinnitus symptoms), 16 patients experienced no change in symptoms, and 1 patient experienced a worsening of tinnitus symptoms. Therefore, a $60 \%$ success rate for MVD of CN VIII was reported. Of the 28 cases for which audiological data were gathered, $60.7 \%$ of patients had varying severities of SNHL. Of the 32 cases for which pre-MVD brainstem auditory evoked response data were gathered, 90.6\% had abnormal findings. 


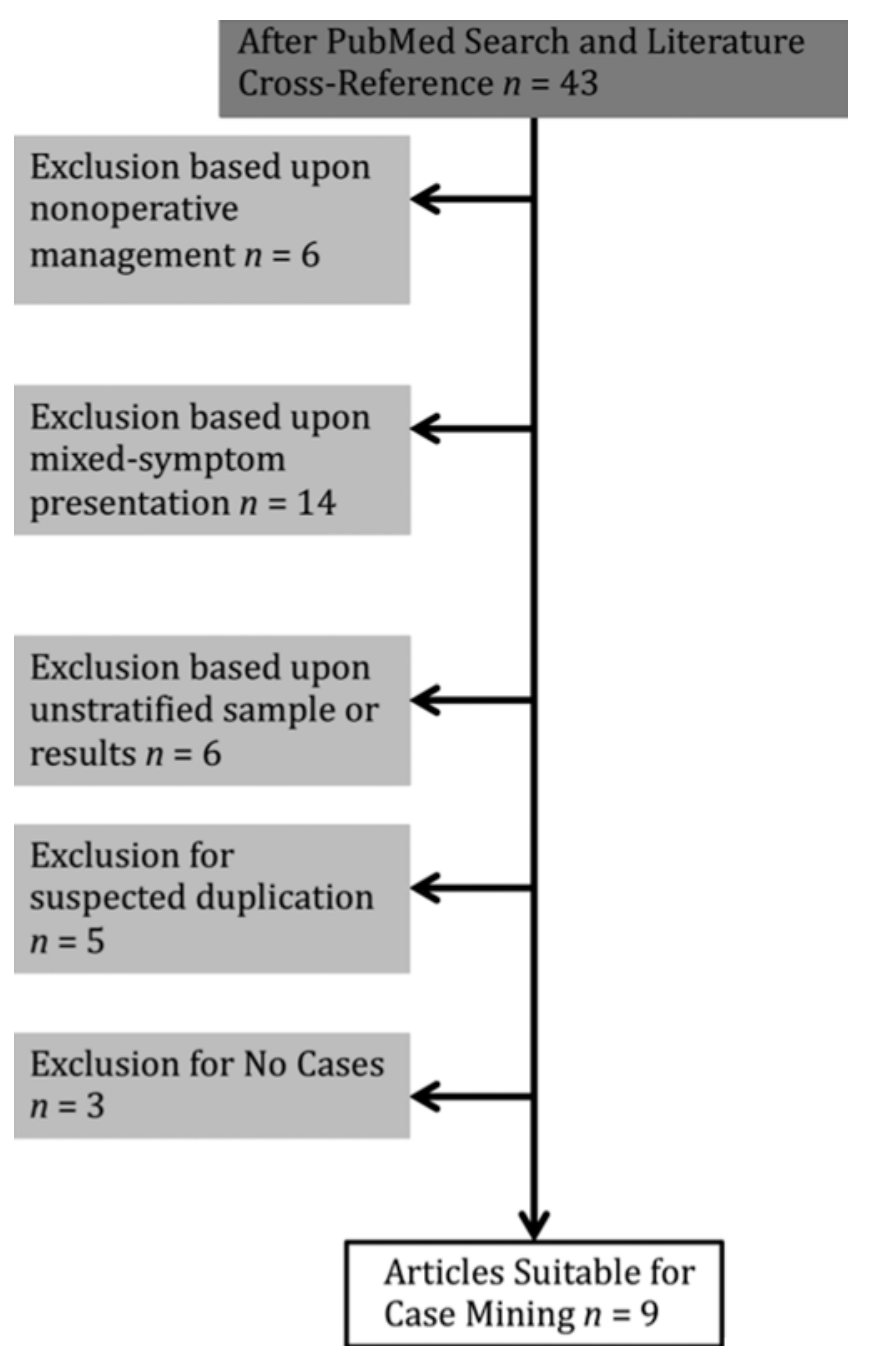

FIG. 1. Flow chart showing tier-one exclusion criteria.

\section{Indications for Surgical Intervention}

Indications for surgical intervention or exploration, especially the role of preoperative imaging, differed between studies. This difference is most striking when examining the literature longitudinally over different eras of imaging technology. For example, of the 9 studies included in the analysis, the 4 earliest publications ${ }^{16,17,22,41}$ and 1 later paper $^{44}$ depended upon clinical presentation as an indication for exploration of the cerebellopontine angle (CPA) over radiological evidence of NVC. Most of these earlier studies $^{16,17,41}$ imaged the CPA with pre-MR modalities such as plain film radiography, air CT, myeloencephalography, and vertebral angiography. These modalities were often "negative" and surgical exploration was undertaken given the patients' great suffering and the lack of an explanation for their tinnitus symptoms. Some of these researchers ${ }^{22,44}$ did perform preoperative MRI on some or all patients but elected exploratory surgery despite negative findings. Later studies ${ }^{5,10,37,46}$ required preoperative radiographic evidence as part of their diagnostic criteria. The difference between these 2 methodologies was explored and it was found that $80 \%$ of patients undergoing CPA explora-

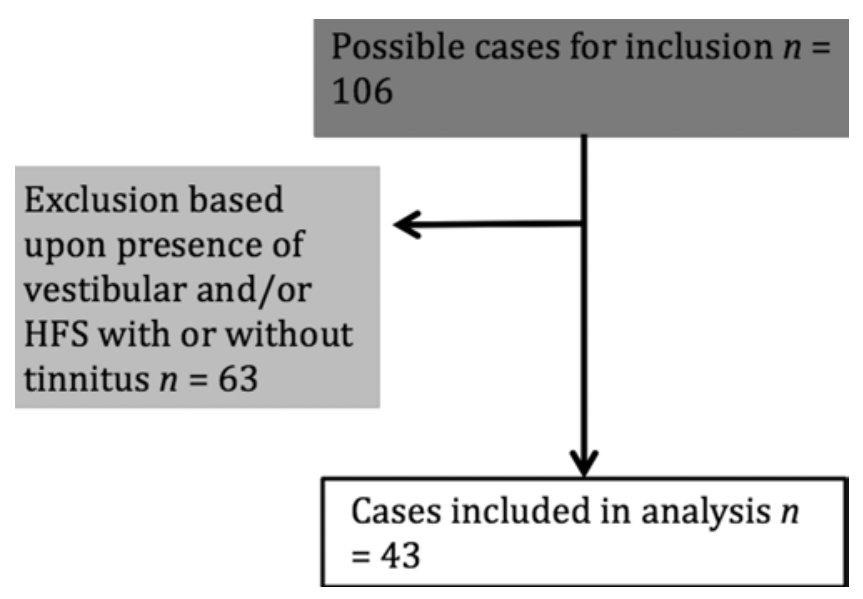

FIG. 2. Flow chart showing tier-two exclusion criteria.

tion, in spite of negative or inconclusive imaging, enjoyed a positive outcome. This is compared with $50 \%$ of patients in studies requiring radiographic evidence of NVC. However, this difference in outcome between the 2 methodologies failed to reach statistical significance upon chi-square analysis $(\mathrm{p}=0.055)$.

\section{Complications}

There were no deaths reported in any of the analyzed studies, but complications did occur. There were $5 \mathrm{CSF}$ leaks reported in the articles of interest. Three leaks required operative repair of damaged mastoid air cells, 1 required lumbar drain placement, and 1 resolved with conservative management. At least 5 patients experienced a decline in hearing postoperatively, with audiometry revealing a mean increase in auditory threshold of $7.9 \mathrm{~dB}$ (range 1-15 dB). The exact number of patients with postoperative hearing decline is unknown as 1 study $^{10}$ presented aggregate SNHL data; however, the mean increase in threshold calculated by Guevara et al. ${ }^{10}$ is included in the above value. Three patients underwent reoperation for treatment failure. Repeat MVDs were attempted in all 3 cases. In 1 of these patients, after the second MVD failed, a third operation was undertaken (neurolysis of CN VIII). Two patients experienced postoperative swallowing difficulty that largely resolved spontaneously. No case included in this review was reported to involve facial paralysis, vertigo/dizziness, meningitis, or stroke as operative complications.

\section{Sex and Outcome}

The relationship between sex and outcome was examined as previous reports have claimed a striking relationship between the two. ${ }^{27}$ Cases $1-26,42$, and 43 were included in the analysis. As 2 cells $(50 \%)$ within the contingency table (Table 2) had expected values less than 5, the continuity correction statistic was consulted in place of the traditional chi-square value. The continuity correction was 0.068 , which failed to reach significance at $\mathrm{p}<0.05$.

\section{Offending Vessel}

Some investigators ${ }^{10}$ have reported differential opera- 


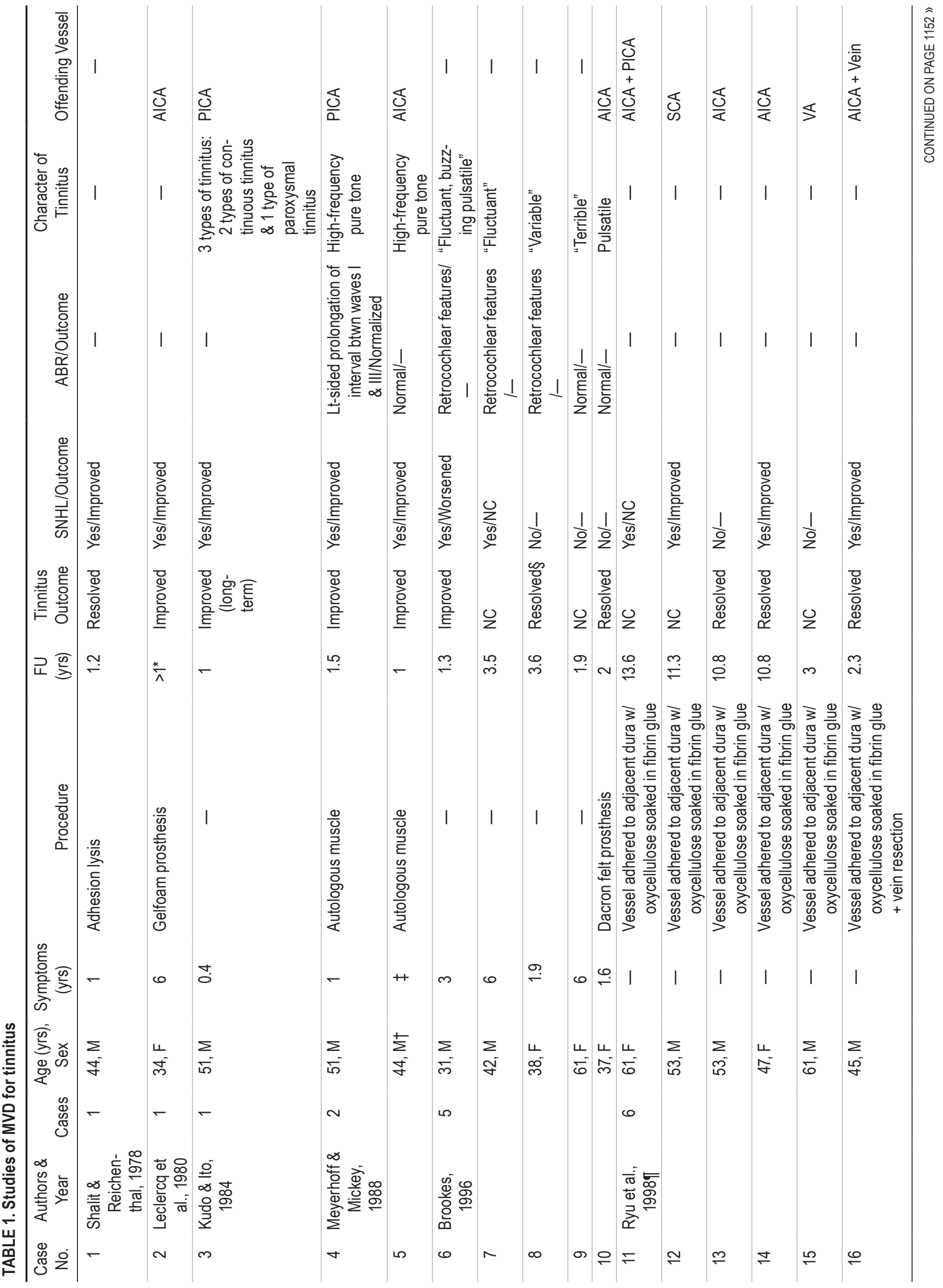




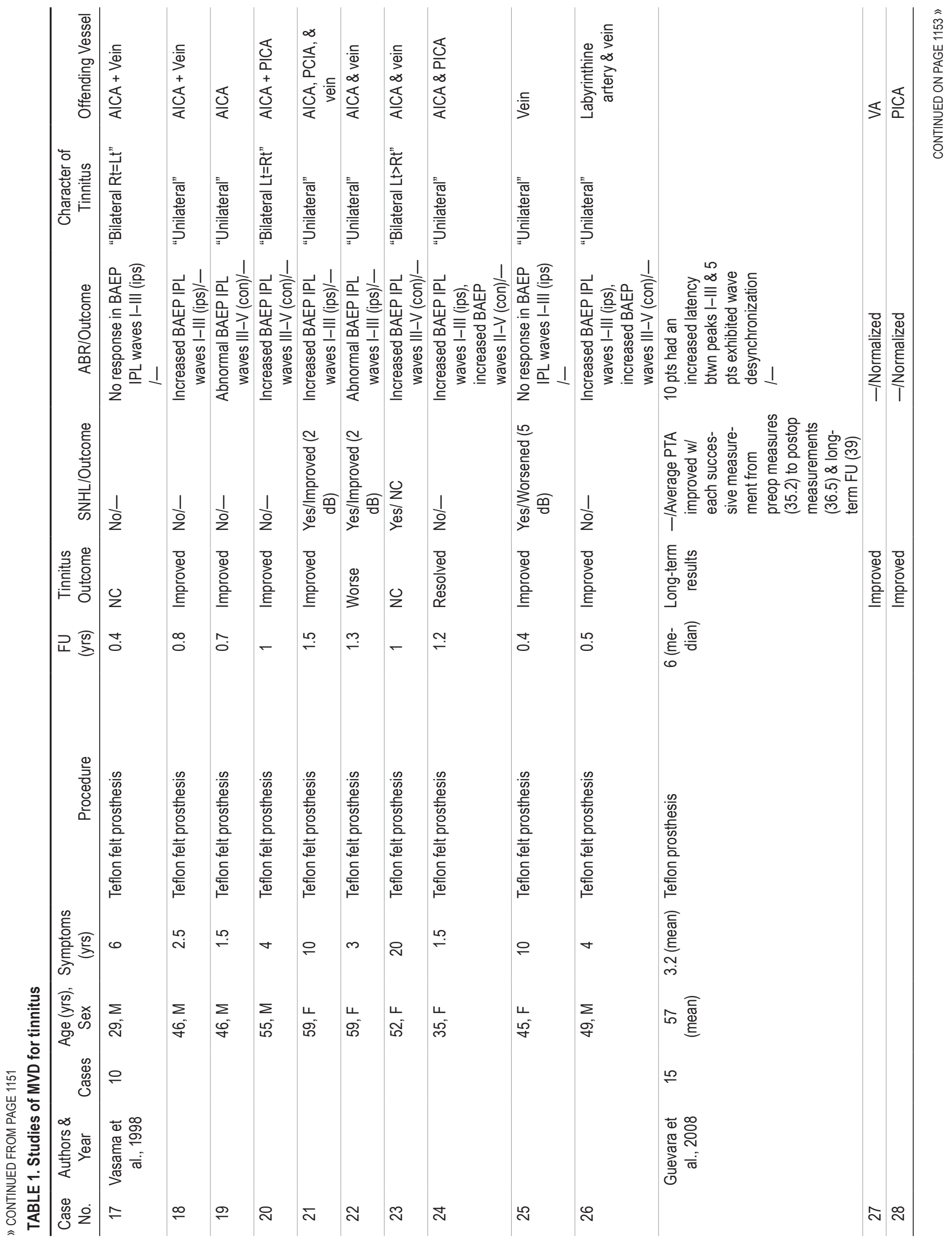




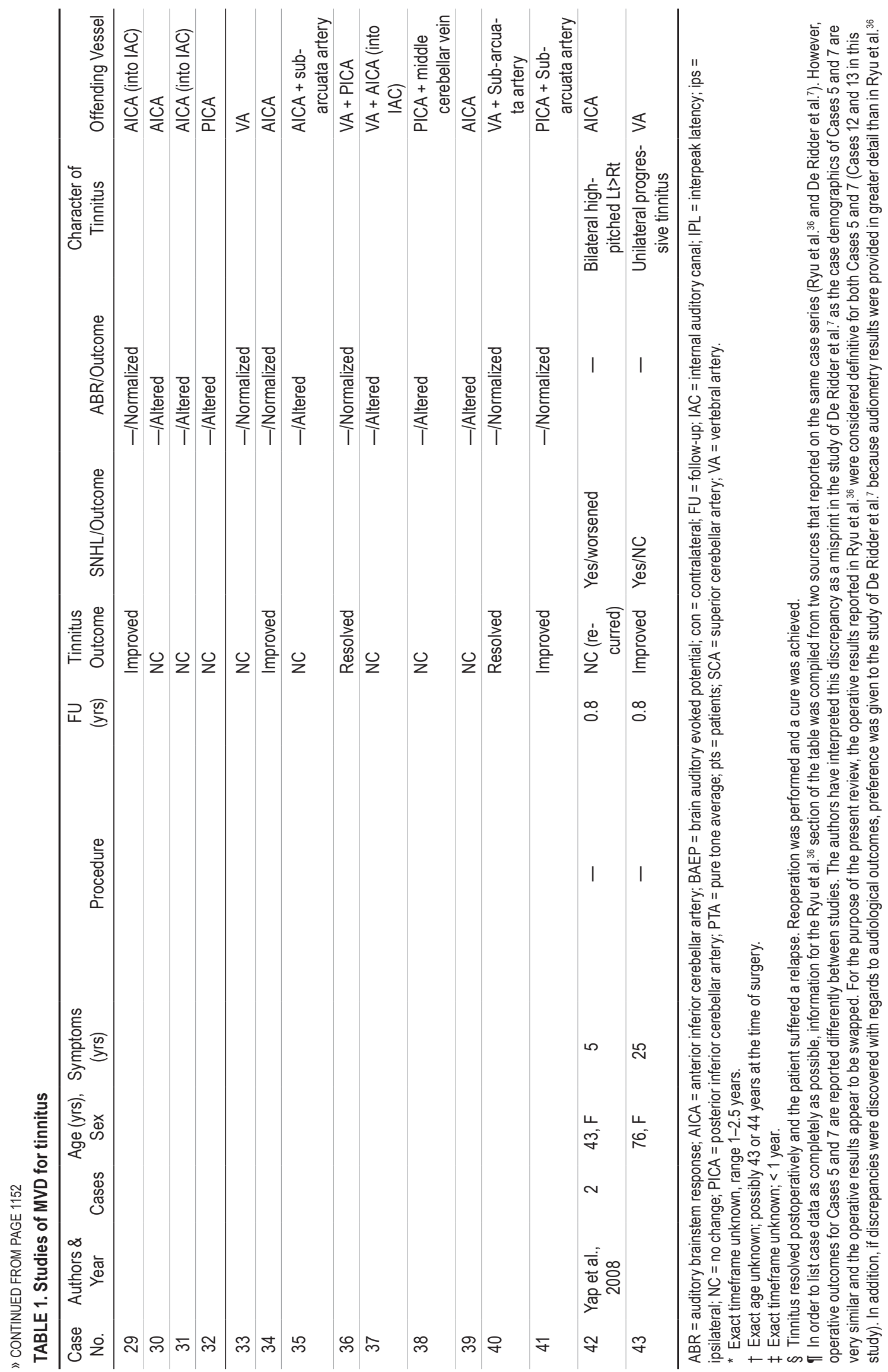


TABLE 2. Contingency table cross-referencing patient sex with operative outcome

\begin{tabular}{|c|c|c|c|}
\hline \multirow[b]{2}{*}{ Sex } & \multicolumn{2}{|c|}{ Outcome } & \multirow[b]{2}{*}{ Total } \\
\hline & Good & Poor & \\
\hline Male & 11 & 4 & 13 \\
\hline Female & 8 & 5 & 15 \\
\hline Total & 19 & 9 & 28 \\
\hline
\end{tabular}

tive results depending upon the culprit vessel compressing CN VIII. To investigate this possibility, Cases 2-5, 10-26, and 27-43 were placed in a contingency table for statistical analysis (Table 3). However, such analysis failed to reach statistical significance $(\mathrm{p}>0.05)$.

\section{Duration of Symptoms}

The duration of symptoms prior to surgery has long been believed to play a role in operative outcome $e^{9,14,27}$ with investigators asserting that the longer the patient suffers from tinnitus the less likely a good operative outcome will occur. Analysis revealed the average preoperative duration of symptoms for those with a good operative outcome to be 3 years compared with an 8-year average for those patients with a poor operative outcome. A cutoff value of 5 years of preoperative symptom duration was determined from the ROC curve. This cutoff value boasts $78.6 \%$ sensitivity and $80 \%$ specificity for a good outcome.

\section{Discussion}

Since Jannetta first introduced MVD for tinnitus, it has remained controversial. This is likely due to the procedure's lower efficacy when compared with MVD for other indications. Based upon the literature, an aggregate success rate of $70 \%$ can be calculated with large variability between studies, ranging from $28 \%$ to $100 \% .5,7,10,15-$ $17,22,27,29,37,39,44,46$ This aggregate rate is lower than the generally accepted $80 \%$ success rate of MVD for TN, which is confirmed in the results of the present study, calculated at a $60 \%$ global success rate for tinnitus-only patients. Again, it may be possible that the difference between the tinnitus-only, literature aggregate, and the MVD for TN rates $-60 \%, 70 \%$, and $80 \%$, respectively-is due in part to the diagnostic dilemma of tinnitus-only patients.

Despite this somewhat lower success rate, these results are still encouraging when compared with other treatment modalities available for tinnitus. As previously noted, the $\mathrm{AAO}-\mathrm{HNS}^{33}$ recommends either hearing aid evaluation or CBT as a starting point for patients with intractable idiopathic tinnitus. CBT does not directly affect tinnitus symptoms but merely helps the patient cope with tinnitusrelated distress. Hearing aids may be helpful in diminishing tinnitus intensity, but this is not considered a method of potential cure. Unlike CBT or hearing aids, a cure has occurred in cases using MVD of CN VIII. In addition, hearing aids are only indicated for those patients with tinnitus and SNHL. Conversely, MVD can be used in cases of tinnitus without SNHL and potentially may address the cause of tinnitus as opposed to only managing the symptom.
TABLE 3. Contingency table cross-referencing offending vessel with operative outcome

\begin{tabular}{ccccccc}
\hline & \multicolumn{5}{c}{ Vessel } & \\
\cline { 2 - 6 } Outcome & AICA & PICA & VA & $\begin{array}{c}\text { Other } \\
\text { Solitary }\end{array}$ & Multiple & Total \\
\hline Good & 8 & 3 & 2 & 1 & 9 & 23 \\
\hline Poor & 4 & 1 & 2 & 1 & 7 & 15 \\
\hline Total & 12 & 4 & 4 & 2 & 16 & 38 \\
\hline
\end{tabular}

Likewise, other operative interventions for tinnitus have been devised, including cochlear implantation, which carries a success rate of $37 \%-61 \%$ for those patients with tinnitus and SNHL.42 However, a potential complication of the procedure is the production of tinnitus in 5\%-12\% of patients. The literature suggests that the risk of tinnitus with MVD is low. Only 1 patient in the current series worsened postoperatively.

Throughout the near 40-year history of the literature on MVD for tinnitus, a number of associations have been drawn between operative outcomes and any multitude of case variables. One of the aims of this study was to compare a select number of those claims from the literature on MVD for tinnitus at large against the tinnitus-only subgroup.

\section{Sex and Outcome}

Møller et al. ${ }^{27}$ reported a difference in positive operative outcome between male and female patients, in which $55 \%$ of female patients experienced a positive outcome compared with $29 \%$ of male patients. This difference, while striking, bears no mention of statistical testing for significance nor has it been replicated in subsequent studies. The authors of the present review wanted to assess tinnitus-only cases for any such sex-outcome relationship. However, none was found.

\section{Offending Vessel}

To the authors' knowledge, Guevara et al. ${ }^{10}$ reported the first and only study to find an association between operative outcome and NVC by a specific vessel. It was discovered that $80 \%$ of patients who suffered CN VIII NVC by the vertebral artery experienced a good operative outcome. No such association between offending vessel and operative outcome was discovered in the tinnitus-only subgroup.

\section{Duration of Symptoms}

The concept that an inverse relationship exists between operative outcomes and preoperative duration of symptoms has been present in the field for decades. It appears to have originated with Jannetta et al., ${ }^{14}$ who argued that reserving surgery for the "worst case"-those most incapacitated by their symptoms - may be a disservice to the patient with tinnitus. Jannetta et al. reasoned that the "worst case" patients most likely had their hyperactive symptoms for a longer preoperative duration, assuming symptoms worsen with time. Jannetta et al. believed that in regards to NVC, CN VIII has some critical period after which 
damage to the nerve may be irreversible and MVD operative outcomes diminished. However, Jannetta et al. did not offer any estimation of this critical period. Møller et al. ${ }^{27}$ made that particular contribution while enumerating the possible reasons MVD of CN VIII has lower success rates than that for other cranial neuralgias. They argued that the point of diminishing returns lies between the 4th and 5 th year of preoperative symptom duration. De Ridder et al. ${ }^{9}$ supported this claim, finding that good outcomes can be obtained with preoperative symptom durations up to 4 years. Corroborating these data, in the present review it was found that among tinnitus-only cases, good outcomes can be predicted with a cutoff of 5 years.

The pathophysiological justification of this "point of diminishing returns" has been addressed in the literature. The suggested mechanism draws upon concepts of the general NVC disease process and electrophysiological studies, specifically in those with CN VIII damage. As has been established for multiple cranial neuralgias, NVC produces progressive damage to CNs. This damage is often defined histologically as demyelination. Some investigators suggest that with increasing demyelination of $\mathrm{CN}$ VIII, afferent information to the cochlear nucleus greatly diminishes or ceases altogether (deafferentation). This loss of sensory input leads to hyperactivity in 1 or more structures belonging to the auditory pathway, which ultimately results in the subjective experience of tinnitus. This central hyperactivity is reflected in observations of increased gamma band activity in the auditory cortex contralateral to CN VIII pathology. ${ }^{5,8-10,44}$

\section{Limitations and Directions for Future Research}

The quality of the present review, like all such articles, is dependent upon the completeness, reliability, and validity of each component study. As the MVD for tinnitus literature spans 4 decades, the articles included in this review are not comparable in every methodological respect. The most glaring difference is the breadth and depth with which case presentations are reported; different authors chose to emphasize different aspects of the case history and workup. As a result of this heterogeneity found in the literature, the present review does not possess a complete data set on every potentially relevant case characteristic.

Based on this incompleteness, several clinical questions remain unanswerable. For example, not every study describes the patient's subjective tinnitus symptoms and those that do provide varying levels of detail. Among cases in which this information was available, patients generally experienced either tonal or pulsatile tinnitus. However, more detailed statements regarding tinnitus pitch or continuity cannot be made as easily. Particular sources provided excellent accounts of case presentations and can serve as templates for future reports including Dandy (1934), ${ }^{6}$ Kudo and Ito (1984), ${ }^{16}$ and Meyerhoff and Mickey (1998)..$^{22}$

In addition, due to the incompleteness with which some cases are indexed, the etiology of tinnitus cannot be guaranteed. There was a concerted effort to exclude from this review any causes of tinnitus not due to NVC. ${ }^{27}$ For example, certain articles were excluded as case data were unstratified by tinnitus etiology. However, given the incon- sistent presentation of case data across the literature it is possible that not all cases ultimately included had the same etiology.

Also, some articles report on patients who had a decidedly vascular character to their presentation, i.e., pulsatile tinnitus corresponding with the patient's heartbeat and/or ceasing with compression of the ipslateral common carotid artery or jugular vein. ${ }^{5,13,18}$ If the presence or absence of this finding were universally reported, this could prove extremely illuminating. In future research, this finding should be correlated with diagnosis and outcome.

Multiple studies present both immediate postoperative results and long-term data. ${ }^{10,47}$ There are obvious differences in outcomes between the two data sets, but no mention is given to the longevity of the procedure. For example, Guevara et al. ${ }^{10}$ report that 10 patients enjoyed a positive outcome in the immediate postoperative period. However, 8 patients had a positive outcome at follow-up. No precise timeframe is given as to the relapse of the 2 missing patients. These data would prove helpful if recorded during future research.

Vasama et al. ${ }^{44}$ found improved outcomes in those with unilateral versus bilateral tinnitus; however, the report of laterality in the literature was not believed to be sufficiently robust to ascertain a correlation with outcome in the present review.

Lastly, inherent to systematic review studies, this review likely suffers from publication bias. It is almost certain that for all the cases reported in the literature there are other unpublished cases for which either no NVC was demonstrated on surgical exploration or MVD did not result in symptom improvement. Thus, there is a strong likelihood that the success rate of $60 \%$ is an overestimation. However, the magnitude of this bias is unknowable.

In an effort to address these and other limitations, the authors encourage the more specific categorization of case presentations as proposed in this review. This would allow for a more thorough analysis of the two different symptom complexes (tinnitus-only and mixed-symptom) and outcomes. In addition, it would aid in collaborative efforts if future studies present case data individually as in the following sources (references 5, 7, 38, and 42).

Some standardization in outcome measures would aid in more valid observations. Prime examples of this include Brookes, ${ }^{5}$ who used audiometric methods to "measure" the loudness of patients' relative tinnitus, and De Ridder et al., ${ }^{9}$ who had each participant rate tinnitus intensity with a visual analog scale score and tinnitus-related distress with a standardized questionnaire.

Furthermore, what is not known is the application of this knowledge toward conditions such as tinnitus associated with acoustic neuroma or other CPA tumors. In patients presenting with disabling tinnitus or vertigo where $\mathrm{CN}$ VIII is preserved in the interest of hearing preservation, very rarely does one perform vascular decompression after removing the tumor. Perhaps this application could be considered in the future.

As previously mentioned, preoperative radiographic visualization of NVC of CN VIII has not been standard practice throughout the history of the MVD procedure. However, the success rates of patients evaluated with and 


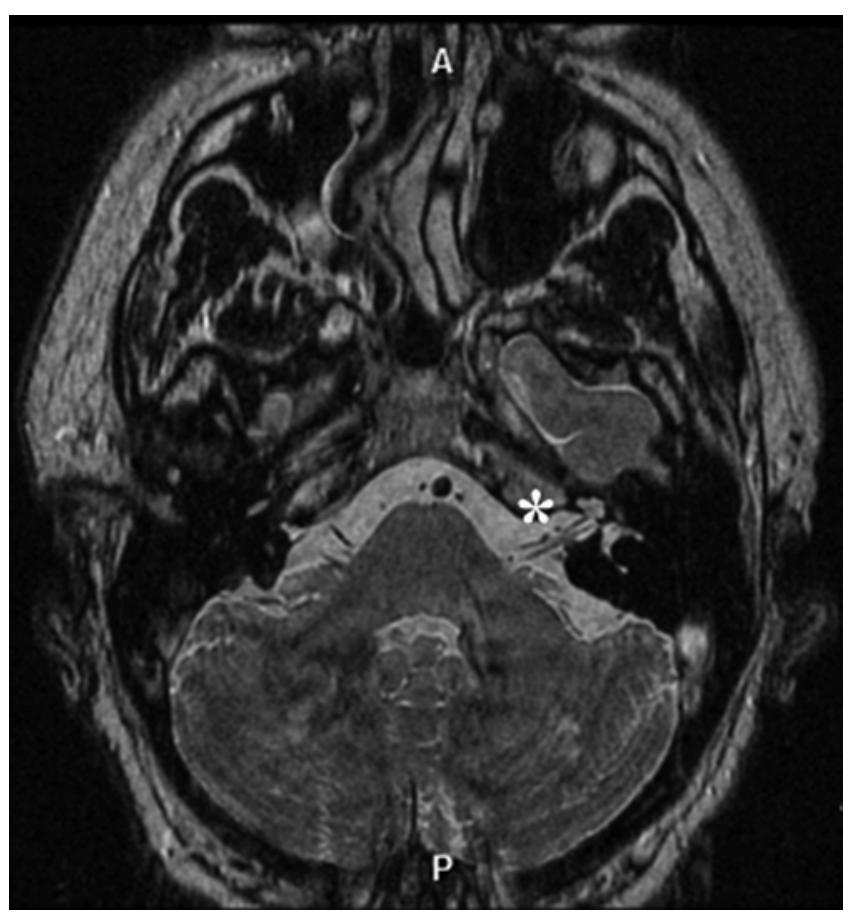

FIG. 3. Axial FIESTA image of probable NVC (asterisk) of CN VIII treated successfully with MVD.

without special emphasis on preoperative imaging showed no difference in surgical outcomes. One would expect lower rates of positive outcomes among patients undergoing exploration without radiographic evidence of NVC. There are numerous possible explanations for why this is not the case, and the primary explanation is publication bias, as discussed earlier. These earlier cases were certainly not the only explorations of the CPA for refractory or unexplained tinnitus taking place, but with the interesting finding of NVC of CN VIII they were published. These cases, then, most likely represent a small number of positive outcomes taken from a larger whole. Considering neuroimaging modalities, no study used newer, more detailed MR sequences, such as fast imaging employing steadystate acquisition (FIESTA) or constructive interference in steady-state. Considering the utility of these sequences in cases of TN, it may be beneficial to explore this highresolution modality with future cases (Fig. 3).

\section{Conclusions}

This review provides the most comprehensive collection of tinnitus-only case data for MVD of CN VIII. An aggregate success rate of MVD for tinnitus was determined to be $70 \%$, with large variability between studies ranging from $28 \%$ to $100 \%$. The success rate of tinnitusonly patients was found to be $60 \%$, possibly reflecting the diagnostic dilemma presented by tinnitus with or without SNHL. The probability of symptom improvement or resolution of tinnitus is greatest in patients reporting a 5-year or less duration of symptoms before operative intervention. The $60 \%$ success rate can be affected both positively and negatively by a number of biases and inconsistencies in the current literature, as outlined above. Thus, further investigation is warranted.

\section{References}

1. Applebaum EL, Valvassori GE: Auditory and vestibular system findings in patients with vascular loops in the internal auditory canal. Ann Otol Rhinol Laryngol Suppl 112:6370,1984

2. Bertrand RA, Molina P, Hardy J: Vestibular syndrome and vascular anomaly in the cerebello-pontine angle. Acta Otolaryngol 83:187-194, 1977

3. Borghei-Razavi H, Darvish O, Schick U: Disabling vertigo and tinnitus caused by intrameatal compression of the anterior inferior cerebellar artery on the vestibulocochlear nerve: a case report, surgical considerations, and review of the literature. J Neurol Surg Rep 75:e47-e51, 2014

4. Brackmann DE, Kesser BW, Day JD: Microvascular decompression of the vestibulocochlear nerve for disabling positional vertigo: the House Ear Clinic experience. Otol Neurotol 22:882-887, 2001

5. Brookes GB: Vascular-decompression surgery for severe tinnitus. Am J Otol 17:569-576, 1996

6. Dandy WE: Concerning the cause of trigeminal neuralgia. Am J Surg 24:447-455, 1934

7. De Ridder D, Ryu H, De Mulder G, Van de Heyning P, Verlooy J, Møller A: Frequency specific hearing improvement in microvascular decompression of the cochlear nerve. Acta Neurochir (Wien) 147:495-501, 501, 2005

8. De Ridder D, Ryu H, Møller AR, Nowé V, Van de Heyning P, Verlooy J: Functional anatomy of the human cochlear nerve and its role in microvascular decompressions for tinnitus. Neurosurgery 54:381-390, 2004

9. De Ridder D, Vanneste S, Adriaenssens I, Lee AP, Plazier M, Menovsky T, et al: Microvascular decompression for tinnitus: significant improvement for tinnitus intensity without improvement for distress. A 4-year limit. Neurosurgery 66:656-660, 2010

10. Guevara N, Deveze A, Buza V, Laffont B, Magnan J: Microvascular decompression of cochlear nerve for tinnitus incapacity: pre-surgical data, surgical analyses and longterm follow-up of 15 patients. Eur Arch Otorhinolaryngol 265:397-401, 2008

11. Jannetta PJ: Neurovascular compression in cranial nerve and systemic disease. Ann Surg 192:518-525, 1980

12. Jannetta PJ: Neurovascular cross-compression in patients with hyperactive dysfunction symptoms of the eighth cranial nerve. Surg Forum 26:467-469, 1975

13. Jannetta PJ: Observations on the etiology of trigeminal neuralgia, hemifacial spasm, acoustic nerve dysfunction and glossopharyngeal neuralgia. Definitive microsurgical treatment and results in 117 patients. Neurochirurgia (Stuttg) 20:145-154, 1977

14. Jannetta PJ, Møller MB, Møller AR, Sekhar LN: Neurosurgical treatment of vertigo by microvascular decompression of the eighth cranial nerve. Clin Neurosurg 33:645-665, 1986

15. Ko Y, Park CW: Microvascular decompression for tinnitus. Stereotact Funct Neurosurg 68:266-269, 1997

16. Kudo T, Ito K: Microvascular decompression of the eighth cranial nerve for disabling tinnitus without vertigo: a case report. Neurosurgery 14:338-340, 1984

17. Leclercq TA, Hill CL, Grisoli F: Retromastoid microsurgical approach to vascular compression of the eighth cranial nerve. Laryngoscope 90:1011-1017, 1980

18. Lesinski SG, Chambers AA, Komray R, Keiser M, Khodadad G: Why not the eighth nerve? Neurovascular compressionprobable cause for pulsatile tinnitus. Otolaryngol Head Neck Surg (1979) 87:89-94, 1979

19. Levine RA: Typewriter tinnitus: a carbamazepine-responsive 
syndrome related to auditory nerve vascular compression. ORL J Otorhinolaryngol Relat Spec 68:43-47, 2006

20. Makins AE, Nikolopoulos TP, Ludman C, O’Donoghue GM: Is there a correlation between vascular loops and unilateral auditory symptoms? Laryngoscope 108:1739-1742, 1998

21. Mathiesen T, Brantberg K: Microvascular decompression for typewriter tinnitus-case report. Acta Neurochir (Wien) 157:333-336, 2015

22. Meyerhoff WL, Mickey BE: Vascular decompression of the cochlear nerve in tinnitus sufferers. Laryngoscope 98:602604, 1988

23. Møller AR: The cranial nerve vascular compression syndrome: I. A review of treatment. Acta Neurochir (Wien) 113:18-23, 1991

24. Møller AR: Vascular compression of cranial nerves. I. History of the microvascular decompression operation. Neurol Res 20:727-731, 1998

25. Møller MB: Results of microvascular decompression of the eighth nerve as treatment for disabling positional vertigo.

Ann Otol Rhinol Laryngol 99:724-729, 1990

26. Møller MB, Møller AR: Audiometric abnormalities in hemifacial spasm. Audiology 24:396-405, 1985

27. Møller MB, Møller AR, Jannetta PJ, Jho HD: Vascular decompression surgery for severe tinnitus: selection criteria and results. Laryngoscope 103:421-427, 1993

28. Nowé V, De Ridder D, Van de Heyning PH, Wang XL, Gielen J, Van Goethem J, et al: Does the location of a vascular loop in the cerebellopontine angle explain pulsatile and nonpulsatile tinnitus? Eur Radiol 14:2282-2289, 2004

29. Okamura T, Kurokawa Y, Ikeda N, Abiko S, Ideguchi M, Watanabe K, et al: Microvascular decompression for cochlear symptoms. J Neurosurg 93:421-426, 2000

30. Ouaknine GE, Robert F, Molina-Negro P, Hardy J: Geniculate neuralgia and audio-vestibular disturbances due to compression of the intermediate and eighth nerves by the postero-inferior cerebellar artery. Surg Neurol 13:147-150, 1980

31. Pirayesh Islamian A, Lütjens G, Krauss JK: Microvascular decompression of the eighth cranial nerve for unilateral pulsatile tinnitus. Clin Neurol Neurosurg 117:102-106, 2014

32. Ramly NA, Roslenda AR, Suraya A, Asma A: Vascular loop in the cerebellopontine angle causing pulsatile tinnitus and headache: a case report. EXCLI J 13:192-196, 2014

33. Rand RW: The Gardner neurovascular decompression operation for trigeminal neuralgia. Acta Neurochir (Wien) 58:161-166, 1981

34. Ryu H, Uemura K, Yokoyama T, Nozue M: Indications and results of neurovascular decompression of the eighth cranial nerve for vertigo, tinnitus and hearing disturbances. Adv Otorhinolaryngol 42:280-283, 1988

35. Ryu H, Yamamoto S: Neurovascular decompression of the eighth cranial nerve for intractable vertigo and tinnitus. Oper Tech Neurosurg 4:142-152, 2001

36. Ryu H, Yamamoto S, Sugiyama K, Nishizawa S, Nozue M: Neurovascular compression syndrome of the eighth cranial nerve. Can the site of compression explain the symptoms? Acta Neurochir (Wien) 141:495-501, 1999

37. Ryu H, Yamamoto S, Sugiyama K, Nozue M: Neurovascular compression syndrome of the eighth cranial nerve. What are the most reliable diagnostic signs? Acta Neurochir (Wien) 140:1279-1286, 1998

38. Ryu H, Yamamoto S, Sugiyama K, Uemura K, Nozue M: Neurovascular decompression of the eighth cranial nerve in patients with hemifacial spasm and incidental tinnitus: an alternative way to study tinnitus. J Neurosurg 88:232-236, 1998

39. Sakaki T, Morimoto T, Miyamoto S, Kyoi K, Utsumi S, Hyo Y: Microsurgical treatment of patients with vestibular and cochlear symptoms. Surg Neurol 27:141-146, 1987

40. Schwaber MK, Hall JW: Cochleovestibular nerve compression syndrome. I. Clinical features and audiovestibular findings. Laryngoscope 102:1020-1029, 1992

41. Shalit MN, Reichenthal E: Anomalous anterior inferior cerebellar artery simulating intracanalicular acoustic nerve tumor. Surg Neurol 10:337-339, 1978

42. Soleymani T, Pieton D, Pezeshkian P, Miller P, Gorgulho AA, Pouratian N, et al: Surgical approaches to tinnitus treatment: A review and novel approaches. Surg Neurol Int 2:154, 2011

43. Tunkel DE, Bauer CA, Sun GH, Rosenfeld RM, Chandrasekhar SS, Cunningham ER Jr, et al: Clinical practice guideline: tinnitus. Otolaryngol Head Neck Surg 151 (2 Suppl):S1-S40, 2014

44. Vasama JP, Moller MB, Moller AR: Microvascular decompression of the cochlear nerve in patients with severe tinnitus. Preoperative findings and operative outcome in 22 patients. Neurol Res 20:242-248, 1998

45. Wuertenberger CJ, Rosahl SK: Vertigo and tinnitus caused by vascular compression of the vestibulocochlear nerve, not intracanalicular vestibular schwannoma: review and case presentation. Skull Base 19:417-424, 2009

46. Yap L, Pothula VB, Lesser T: Microvascular decompression of cochleovestibular nerve. Eur Arch Otorhinolaryngol 265:861-869, 2008

47. Zhang L, Yu Y, Yuan Y, Xu J, Xu X, Zhang J: Microvascular decompression of cochleovestibular nerve in patients with tinnitus and vertigo. Neurol India 60:495-497, 2012

\section{Disclosures}

The authors report no conflict of interest concerning the materials or methods used in this study or the findings specified in this paper.

\section{Author Contributions}

Conception and design: Van Gompel, Carlson. Acquisition of data: Nash. Analysis and interpretation of data: Nash. Drafting the article: Nash. Critically revising the article: Van Gompel, Carlson. Reviewed submitted version of manuscript: all authors. Study supervision: Van Gompel, Carlson.

\section{Correspondence}

Jamie J. Van Gompel, Department of Neurological Surgery, Mayo Clinic, 200 First St. SW, Rochester, MN 55905. email: vangompel.jamie@mayo.edu. 